\title{
Statistical Enhancement of Terminal Current Estimation for Monte Carlo Device Simulation
}

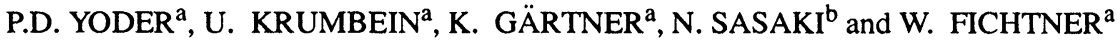 \\ ${ }^{\mathrm{a}}$ Swiss Federal Institute of Technology, Integrated Systems Laboratory, Gloriastrasse 35, CH-8092 Zürich, Switzerland, \\ ${ }^{\mathrm{b}}$ Fujitsu Laboratories, Ltd., ULSI Research Division Atsugi 243-01, Japan
}

\begin{abstract}
We present a new generalized Ramo-Shockley theorem (GRST) to evaluate contact currents, applicable to classical moment-based simulation techniques, as well as semiclassical Monte Carlo and quantum mechanical transport simulation, which remains valid for inhomogeneous media, explicitly accounts for generation/recombination processes, and clearly distinguishes between electron, hole, and displacement current contributions to contact current. We then show how this formalism may be applied to Monte Carlo simulation to obtain equations for minimum-variance estimators of steady-state contact current, making use of information gathered from all particles within the device. Finally, by means of an example, we demonstrate this technique's performance in acceleration of convergence time.
\end{abstract}

Keywords: Monte Carlo simulation, Terminal current calculation, Generalized Ramo-Shockley theorem

\section{INTRODUCTION: THE RAMO-SHOCKLEY THEOREM}

Through application of the method of Green functions in the quasi-electrostatic approximation, Shockley [1] and Ramo [2] introduced the original domain integration formula relating the currents induced on an arbitrary number of contacts to the motion of charges in multiple dimensions :

$$
I^{(k)}=\sum_{j} q_{j} \mathbf{E}_{j}^{(k)} \cdot \mathbf{v}_{j}
$$

where the index $k$ indicates the contact at which the current is to be evaluated, $q_{j}$ and $\mathbf{v}_{j}$ represent particle charge and velocity, respectively, and the index $j$ runs over all particles within the volume. The symbol $\boldsymbol{E}_{j}^{(k)}$ denotes the electric field at the position of particle $j$ which would result if all charges were removed from the volume, and boundary conditions were imposed such that all contacts are grounded with the exception of contact $k$, which is set to 1 Volt.

\section{A GENERALIZED RAMO-SHOCKLEY THEOREM}

One of the most fundamental properties of all types of transport are the continuity equations, which may be derived by taking moments of the appropriate transport equations. For classical and semi-classical systems, this is most often the Boltzmann Transport equation [3], and either the Wigner-Boltzmann equation [4] or the quantum Liouville equation [5] for quantum systems. The $0^{\text {th }}$ moments take on the following general form:

$$
\begin{aligned}
-\nabla \cdot \mathbf{j}_{n} & =e G_{n}(\mathbf{r})-e \dot{n}, \\
-\nabla \cdot \mathbf{j}_{p} & =e G_{p}(\mathbf{r})+e \dot{p},
\end{aligned}
$$


Furthermore, Maxwell's equations may be used to derive equally familiar identities for the displacement current $\mathbf{j}_{d}$ and total current $\mathbf{j}_{t}$ :

$$
\begin{aligned}
-\nabla \cdot \mathbf{j}_{d}=\nabla \cdot\left(\mathbf{j}_{n}+\mathbf{j}_{p}\right) & =-e(\dot{p}+\dot{n}) \\
-\nabla \cdot \mathbf{j}_{t} & =0,
\end{aligned}
$$

where the variables $n$ and $p$ refer to the electron and hole free carrier densities, respectively. Let $h_{k l}$ be a suitable set of test functions satisfying

$$
\left.h_{k l}\right|_{\Gamma_{l}}=1,\left.\quad h_{k l}\right|_{\Gamma_{j}}=0, \quad j \neq l, \quad k=n, p, d, t,
$$

where $\Gamma$ denotes the boundary, consisting of both Neumann $\left(\Gamma_{N}\right)$ and Dirichlet $\left(\Gamma_{D}\right)$ parts, and $\Gamma_{D}=\cup_{i} \Gamma_{i}$. Upon multiplication of an equation of the form $-\nabla \cdot \mathbf{j}_{k}=R_{k}$ with a test function $h_{k}$, integration by parts yields $[6,7]$ :

$$
\begin{gathered}
\left(-\nabla \cdot \mathbf{j}_{k}, h_{k l}\right)=\left(R_{k}, h_{k l}\right), \\
-\int_{\Omega} h_{k l} \nabla \cdot \mathbf{j}_{k} d V=-\int_{\Gamma} h_{k l} \mathbf{j}_{k} d \mathbf{S}+\int_{\Omega} \nabla h_{k l} \cdot \mathbf{j}_{k} d V .
\end{gathered}
$$

The definition of $h_{k l}$ requires that the surface integral is equivalent to the contact current, because $h_{k l \mid \Gamma_{m}}=\delta_{l m}$ and $\left.\left(\boldsymbol{j}_{k} \cdot \hat{\boldsymbol{n}}\right)\right|_{\Gamma_{N}}=0$. Equations (2), (3), (4), (5), and (7), lead to the following formulas for the terminal currents at an arbitrary contact $l$ :

$$
\begin{aligned}
& I_{n l}=\int \nabla h_{n l}(\mathbf{r}) \cdot \mathbf{j}_{n} d V+e \int \dot{n} h_{n l}(\mathbf{r}) d V \\
& -e \int G_{n}(\mathbf{r}) h_{n l}(\mathbf{r}) d V \\
& I_{p l}=\int \nabla h_{p l}(\mathbf{r}) \cdot \mathbf{j}_{p} d V-e \int \dot{p} h_{p l}(\mathbf{r}) d V \\
& +e \int G_{p}(\mathbf{r}) h_{p l}(\mathbf{r}) d V, \\
& I_{d l}=\int \nabla h_{d l}(\mathbf{r}) \cdot \mathbf{j}_{d} d V-\int h_{d l}(\mathbf{r}) \nabla \cdot\left(\mathbf{j}_{n}+\mathbf{j}_{p}\right) d V \\
& =\int \nabla h_{d l}(\mathbf{r}) \cdot \mathbf{j}_{d} d V+e \int(\dot{p}-\dot{n}) h_{d l}(\mathbf{r}) d V, \\
& I_{t l}=\int \nabla h_{t l}(\mathbf{r}) \cdot\left(\mathbf{j}_{n}+\mathbf{j}_{p}+\mathbf{j}_{d}\right) d V .
\end{aligned}
$$

This method is also applicable to terminal currents of quantities derived from higher-order moments of the carrier distribution functions, inhomogeneous media and high frequencies [8]. The steady-state form of these equations is obtained by setting all time derivatives equal to zero.

\section{OPTIMIZED STEADY-STATE FORM OF THE GRST}

Convergence time for steady-state particle-based simulation is limited by estimator variance. Minimizing the functional form of the steady-state terminal current estimator variances $\left\langle\left(\hat{I}_{k l}-\bar{I}_{k l}\right)^{2}\right\rangle$ with respect to the test functions $h_{k}$, under the assumption that fluctuations in particle trajectories about the spatiallydependent mean trajectory have only weak interparticle correlation, results in the following set of EulerLagrange equations :

$$
\begin{gathered}
\nabla \cdot\left\langle\delta \hat{\mathbf{j}}_{k} \otimes \delta \hat{\mathbf{j}}_{k}\right\rangle \cdot \nabla h_{k l}^{o p t}(\mathbf{r})=0 \\
\left.h_{k l}^{o p t}\right|_{\Gamma_{m}}=\delta_{l m}, \quad h \in H^{1}, \quad k=n, p, t .
\end{gathered}
$$

The quantity $\langle\delta \hat{j} \otimes \delta \hat{j}\rangle$ is the position-dependent current density variance tensor. In steady-state simulation, it has the following numerically convenient tensor and scalar approximations

$$
\begin{gathered}
\left\langle\hat{\mathbf{j}}_{k} \otimes \hat{\mathbf{j}}_{k}\right\rangle_{i i} \approx\left(\rho_{k}\right)^{2}\left(v_{T, k, i}^{2}-\bar{v}_{k, i}^{2}\right) \tau_{c, k} /\left(N_{k} t\right), \\
\left\|\left\langle\mathbf{j}_{k} \otimes \mathbf{j}_{k}\right\rangle\right\| \approx\left(\rho_{k}\right)^{2}\left(v_{T, k}^{2}-\bar{v}_{k}^{2}\right) \tau_{c, k} /\left(N_{k} t\right),
\end{gathered}
$$

where the symbols $\rho_{k}, v_{T, k}$ and $\bar{v}_{k}$ are the charge density, thermal velocity and mean velocity, respectively, for particles of type $k . N_{k}$ and $\tau_{c, k}$ are the grid-dependent mean type- $k$ particle number and momentum relaxation time, and $t$ is the simulation time. The corresponding terminal current estimators then become:

$$
\begin{gathered}
\hat{I}_{n l}=\int \nabla h_{n l}^{o p t}(\mathbf{r}) \cdot \mathbf{j}_{n} d V-e \int G_{n}(\mathbf{r}) h_{n l}^{o p t}(\mathbf{r}) d V, \\
\hat{I}_{p l}=\int \nabla h_{p l}^{o p t}(\mathbf{r}) \cdot \mathbf{j}_{p} d V+e \int G_{p}(\mathbf{r}) h_{p l}^{o p t}(\mathbf{r}) d V, \\
\hat{I}_{t l}=\int \nabla h_{t l}^{o p t}(\mathbf{r}) \cdot\left(\mathbf{j}_{n}+\mathbf{j}_{p}\right) d V .
\end{gathered}
$$

\section{APPLICATION TO MOSFET SIMULATION}

To extract current-voltage characteristics of an $0.5 \mu \mathrm{m}$ n-Mosfet device, several simulations were performed with the Monte Carlo simulator Degas. Test functions for the terminal current estimators were computed 

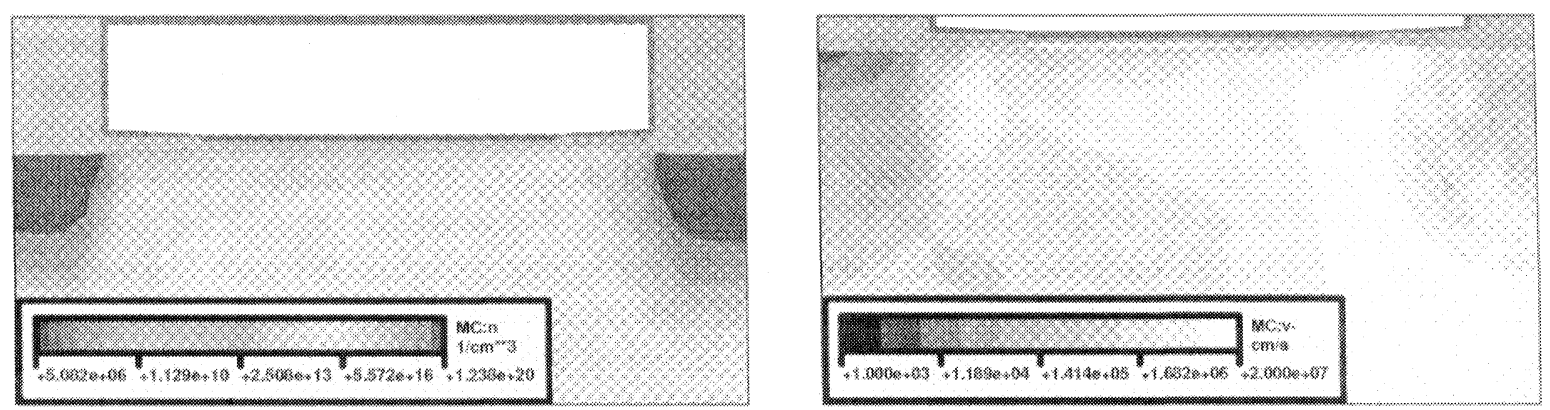

FIGURE 1 Simulated electron density and velocity profiles for a 0.5 micron MOSFET. $V_{g s}=2 \mathrm{~V}, V_{d s}=4.875 \mathrm{~V}$
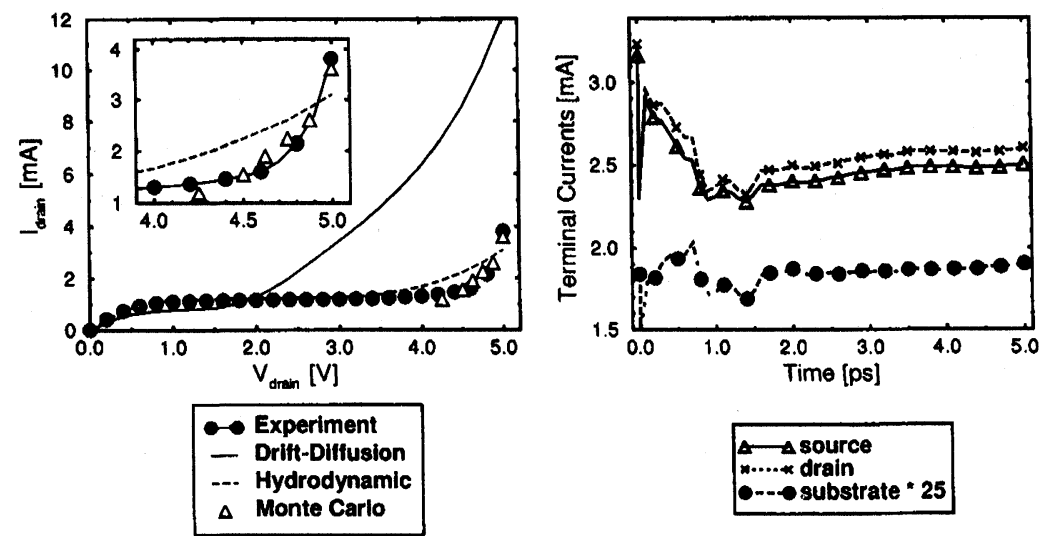

FIGURE 2 Simulated and experimental $I_{d}-V_{d s}$ characteristics, along with convergence times for source, drain and substrate currents at $V_{d s}=4.875 \mathrm{~V}$ and $V_{g s}=2 \mathrm{~V}$

from equations (12), (15) and (16), and used along with simulated carrier density and velocity profiles, such as shown in Fig. 1, to obtain the cumulatively time-averaged steady-state drain currents shown in Fig. 2. All simulations were run with 20000 electrons and 10000 holes. In comparison to the particle counting technique, convergence is reached with the optimzed GRST method between 10 and 40 times faster for these examples.

\section{Acknowledgements}

The authors are grateful to H. Gotoh, Fujitsu Limited, Kawasaki, for providing experimental data.

\section{References}

[1] W. Shockley, Journal of Applied Physics, 9, 635 (1938).

[2] S. Ramo, Proceedings of the IRE, 27, 584 (1939)

[3] K. Hess, Advanced Theory of Semiconductor Devices, Prentice Hall, Englewood Cliffs, New Jersey (1988).
[4] C. L. Gardner, SIAM Journal on Applied Mathematics 54, 409 (1994).

[5] H. L. Grubin, T. R. Govindan, J. P. Kreskovsky, and M. A. Stroscio, Solid-State Electronics, 36, 1697 (1993).

[6] M. S. Mock, Analysis of Mathematical Models of Semiconductor Devices, Boole Press Limited, Dublin, Ireland 1983.

[7] A. Gajewski, private communications, ca. 1985.

[8] P. D. Yoder, K. Gärtner and W. Fichtner, Journal of Applied Physics, 79, 1951 (1996).

\section{Biographies}

P. D. Yoder was born in Washington, D.C., on February 15,1968 . He received the B.S.E.E. degree from Cornell University, Ithaca, NY, in 1989, and the M.S. and $\mathrm{Ph} . \mathrm{D}$. degrees in electrical engineering from the University of Illinois, Urbana, IL, in 1991 and 1993, respectively. Since graduation he has been employed at the Integrated Systems Laboratory of the Swiss Federal Institute of Technology (ETH) in Zurich, Switzerland, working in the area of silicon devices. 
His professional interests include semiconductor device physics, applied mathematics, and Monte Carlo simulation.

Ulrich Krumbein was born in Braunschweig, Germany in 1964. In 1990 he recieved the Diploma in physics from the University of Kaiserslautern, Germany. In 1991 he joined the Integrated Systems Laboratory at the ETH in Zurich, Switzerland, where he is currently working towards his Ph.D. in semiconductor device simulation. His research interests are in physical models for silicon device simulation, with special emphasis on EEPROM devices.

K. Gärtner was born in Zittau, Germany, on March 4, 1950. He studied theoretical physics in Dresden (Germany), and received a $\mathrm{PhD}$ in nuclear reactor physics. After working ten years in the field of neutron transport, he joined the Karl-Weierstraß-Institute for Mathematics in Berlin in 1982. Since this time, his main interest has been in numerical problems connected to PDE's and semiconductor device models. In 1992, he joined the Interdisciplinary Center for Supercomputering at the ETH, and since 1994 has been working with Prof. Fichtner at the ETH Integrated Systems Lab.

Nobuo Sasaki was born in Matsuyama, Japan, in 1949. He received the B.S. degree in Physics and Ph.D. degree in Electrical Engineering in 1971 and
1984, respectively, both from the University of Tokyo, Japan. He joined Fujitsu Limited, Kawasaki, in 1971. He moved to Fujitsu Laboratories, Atsugi, in 1993, where he is currently a director of ULSI Technology Laboratory. He has studied device physics of MOSFET's, 3-dimensional integrations, and electrically conducting polymers. His main interest is now in Gbit-DRAM's. In 1985, he received the award of Minister of Science and Technology Agency for his contributions to the SOI technology and 3-dimensional integrations.

Wolfgang Fichtner received the Dipl. Ing. degree in physics and the Ph.D. degree in electrical engineering from the Technical University of Vienna, Austria, in 1974 and 1978, respectively. From 1975 to 1978, he was an Assistant Professor in the Department of Electrical Engineering, Technical University of Vienna. From 1979 through 1985, he worked at AT\&T Bell Laboratories, Murray Hill, NJ. Since 1985 he is Professor and Head of the Integrated Systems Laboratory at the Swiss Federal Institute of Technology (ETH). In 1993, he founded ISE Integrated Systems Engineering AG, a company in the field of technology CAD. Wolfgang Fichtner is also a fellow of the IEEE and a member of the Swiss National Academy of Engineering. 

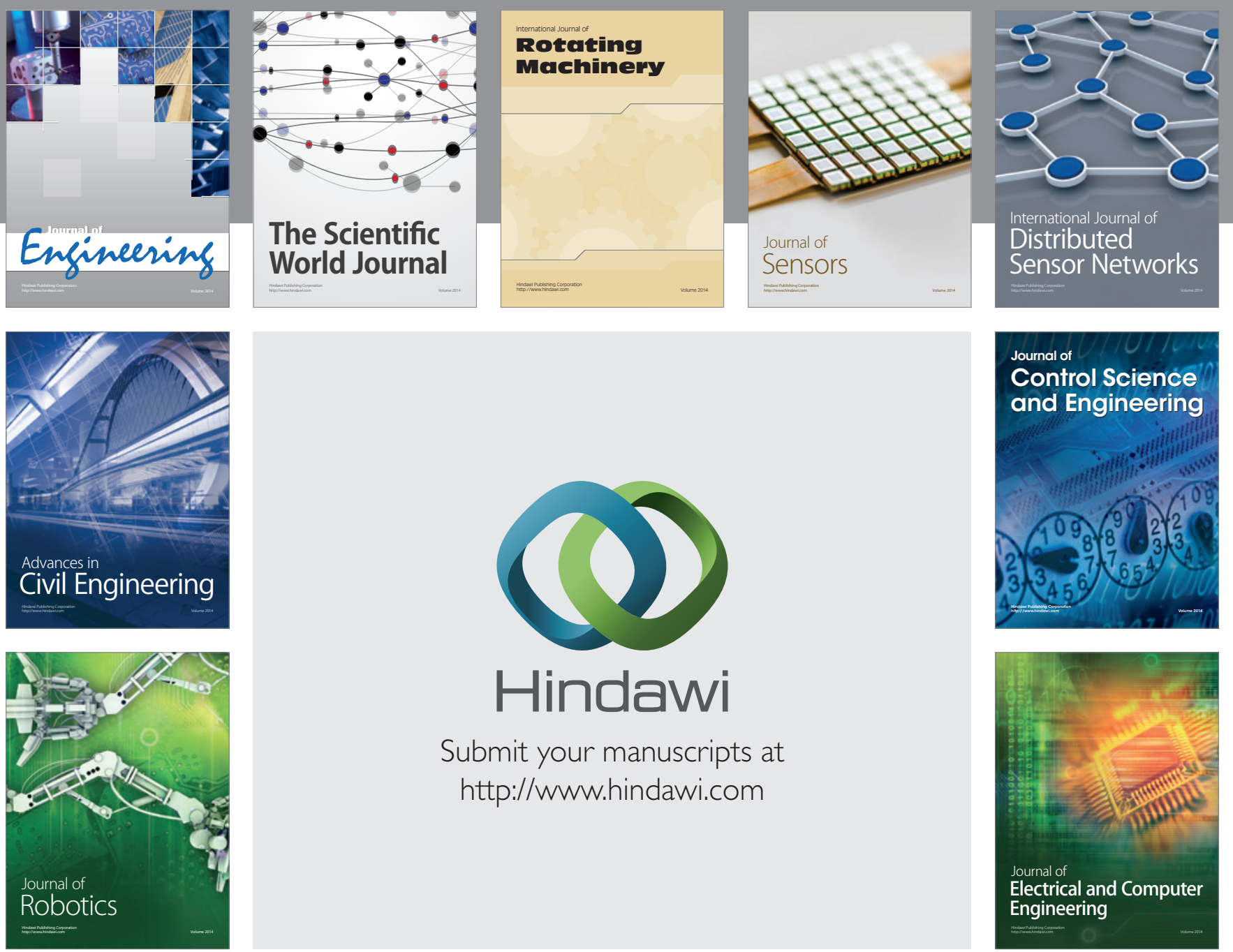

Submit your manuscripts at

http://www.hindawi.com
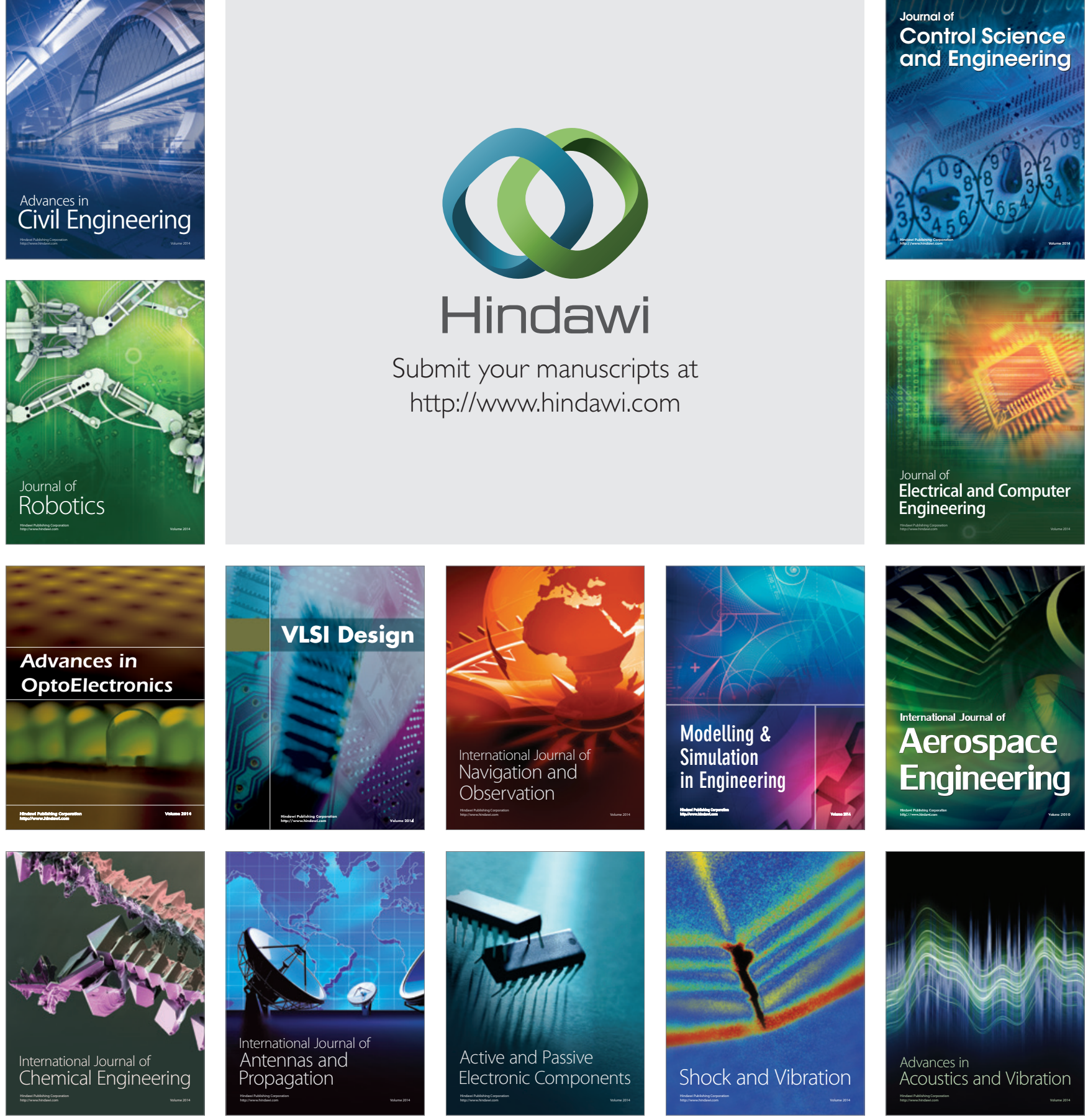